\title{
Temporal trends in healthcare resource utilization and costs following acute myocardial infarction
}

\author{
Arthur Shiyovich ${ }^{1,2^{*}}$, Harel Gilutz ${ }^{3}$, Jonathan Eli Arbelle ${ }^{3,4}$, Dan Greenberg ${ }^{3}$ and Ygal Plakht Hats $^{3,5}$
}

\begin{abstract}
Background: Acute myocardial infarction (AMI) is associated with greater utilization of healthcare resources and financial expenditure.

Objectives: To evaluate temporal trends in healthcare resource utilization and costs following AMl throughout 2003-2015.

Methods: AMI patients who survived the first year following hospitalization in a tertiary medical center (Soroka University Medical Center) throughout 2002-2012 were included and followed until 2015. Length of the in-hospital stay (LOS), emergency department (ED), primary care, outpatient consulting clinic visits and other ambulatory services, and their costs, were evaluated and compared annually over time.

Results: Overall 8047 patients qualified for the current study; mean age 65.0 (SD = 13.6) years, 30.3\% women. During follow-up, LOS and the number of primary care visits has decreased significantly. However, ED and consultant visits as well as ambulatory-services utilization has increased. Total costs have decreased throughout this period. Multivariate analysis, adjusted for potential confounders, showed as significant trend of decrease in LOS and ambulatory-services utilization, yet an increase in ED visits with no change in total costs.
\end{abstract}

Conclusions: Despite a decline in utilization of most healthcare services throughout the investigated decade, healthcare expenditure has not changed. Further evaluation of the cost-effectiveness of long-term resource allocation following AMl is warranted. Nevertheless, we believe more intense ambulatory follow-up focusing on secondary prevention and early detection, as well as high-quality outpatient chest pain unit are warranted.

Keywords: Acute myocardial infarction; healthcare resource utilization, Costs, Temporal trends

\section{Introduction}

Survivors of acute myocardial infarction (AMI) continue to be at greater risk and utilize more healthcare resources, resulting in increased economic burden [1-3]. Moreover, expenditure throughout the first year following an AMI seems to be exceptionally high, estimated between $\$ 22,000-\$ 87,000$ (European countries and the United States) [3-6]. Most aspects of AMI management have experienced dramatic changes throughout recent years, including patient characteristics, presentation,

\footnotetext{
* Correspondence: arthur.shiyovich@gmail.com

${ }^{1}$ Department of Cardiology, Beilinson Hospital, Rabin Medical Center, Rabin Medical Center, 39 Jabotinski Street, 49100 Petah Tikva, Israel

${ }^{2}$ Sackler Faculty of Medicine, Tel Aviv University, Tel Aviv, Israel

Full list of author information is available at the end of the article
}

invasive procedures, medical therapy, and outcomes which overall have improved significantly $[1,7]$. However, data dealing with healthcare resource utilization and expenditure are sparse and inconsistent $[8,9]$. The aim of the current study was to evaluate the temporal trends in healthcare resource utilization and costs following AMI.

\section{Methods}

Study population

The current study included patients who survived the first year following hospitalization with an AMI (index admission) throughout 1.1.2002-31.12.2012 in Soroka University Medical Center and who were members of one of the two largest insurers in Israel (Health 
Maintenance Organizations - HMOs): Maccabi Healthcare Services or (Maccabi) or Clalit Health Services (Clalit). For patients who suffered more than one AMI throughout the investigated period, the first event was considered as the index event.

Soroka University Medical Center is a tertiary referral center ( 1200 beds), serving the metropolitan area of Beer-Sheva, Negev, southern Israel. This region comprises approximately $60 \%$ of the area of Israel and is inhabited by more than 500,000 residents, Jews over $60 \%$. Thus, this hospital and its cardiology department face unique challenges: for example management of acute myocardial infarction transferred after thrombolytic therapy in Eilat (the most southern city in Israel), while all other cardiology departments in Israel can provide primary PCI services for STEMI.

The local ethics committees of the participating organizations approved the study, which was performed consistently with the Helsinki declaration.

\section{Individual follow-up and study outcomes}

The personal follow-up of the study participants began 1 year after the index admission (i.e. 1 year survivors) and lasted up to10 years (or until the participant's death). The follow-up period of the study ended in 31.12.2015. Evaluation of healthcare services utilization comprised the following: the length of in-hospital stay, all causes, number of emergency department (ED) visits not resulting in hospital admissions, number of primary care and outpatient clinic visits and other ambulatory services (e.g. various ambulatory diagnostic or therapeutic procedures). In addition, the per-patient total cost was estimated by summing the costs of all the utilized services.

\section{Data sources and classifications}

Data were retrieved from the participating organizations' computerized medical records. The baseline data obtained from the hospitals' database included patient characteristics clinical evaluation and management as previously reported for the Soroka Acute Myocardial Infarction (SAMI-II) project [10]. Most variables were obtained using The International Classification of Diseases, Ninth Revision, Clinical Modification (ICD-9-CM) discharge codes (see Appendix for examples). Furthermore, the diagnosis of anemia was grouped together with low hematocrit and low hemoglobin blood levels at discharge as following: for men-hemoglobin $<13 \mathrm{~g} / \mathrm{dL}$ and hematocrit $<39 \%$; for women-hemoglobin $<12 \mathrm{~g} /$ $\mathrm{dL}$ and hematocrit $<36 \%$. Similarly, the diagnosis of renal diseases included high creatinine blood levels (at discharge) $\geq 1.2 \mathrm{mg} / \mathrm{dL}$. The diagnosis of diabetes mellitus comprised high levels of Hemoglobin A1C (greater than 6.5\%). Diagnoses of diabetes mellitus (DM) with complications were classified in accordance with the target of complications. In this way, the diagnoses of DM with renal manifestations were defined as renal diseases, the diagnoses of DM with peripheral circulation manifestations were grouped with peripheral vascular diseases. The remaining diagnoses of DM were assigned to DM with no recorded complications.

Adherence with medical therapy for the following guideline-recommended medications: Aspirin, statins, beta blockers and angiotensin-converting-enzyme inhibitors (ACEi) or Angiotensin II receptor blockers (ARBs). The adherence rate was calculated based on the issued prescriptions during the first year following hospital discharge and calculated consistently with the well described proportion of days covered (PDC) parameter [11]. Adherence (PDC) of $80 \%$ or more for all the evaluated medication groups was considered as adherent [12-15].

Utilization of the various healthcare services were obtained from the records of the insurers. Per-patient costs were obtained for the year before the index admission and for each year throughout the follow-up period thereafter. Costs were based on the rates set by the Israeli Ministry of Health. Costs were obtained in local currency (Israeli Shekel - [ILS]) and converted to United States Dollar (USD) based on currency exchange rates (1 ILS $=0.29$ USD, 04.01.2020). In this context it should be mentioned, that primary clinic visits mainly refers to a physician-patient encounter while ambulatory visit includes various ambulatory procedures (analyses or treatments) which are charged differently by the HMOs.

Mortality data were obtained from the Ministry of the Interior Population Registry.

Patient-level data from the records of the abovementioned authorities were linked through the individual personal identification number.

\section{Statistical analysis}

Statistical analyses were performed with IBM SPSS Statistics 25 software. Baseline characteristics of the study cohort were presented as mean and standard deviation (SD) for continuous variables and $\mathrm{n} /$ percent for categorical data. Healthcare service utilization and their costs were calculated for every year throughout 2003-2015 and are presented as mean and SD. Comparisons of the investigated outcomes between the years throughout the study period were performed using Analysis of variance (ANOVA) test for linear trend in the univariate level. In addition, generalized estimating equations (GEE) were built separately for each investigated outcome, for the purpose of controlling for repeated measures for each patient. Three level models for each outcome were built. The first level model ("unadjusted") included the variable of the study year only. The second level model included the variable of the study year and the personal time following the AMI (follow-up period). The third level 
model included all the above variables in addition to the patients' baseline characteristics, healthcare service utilization 1 year before AMI and adherence with medical therapy during the first year after AMI. The results of the models are presented as the coefficients (B) with Standard errors (SE) and 95\% confidence intervals (CI). For each test, two-sided $p<0.05$ was considered significant.

\section{Results}

\section{Baseline characteristics}

Throughout 2002-2012 a total of 12,535 patients were admitted with an AMI and screened for the current study. Overall 8047 patients qualified for the study (mean age $64.96 \pm 13.58$ ), as the rest were excluded for the following reasons: 2293 (18.3\%) were not insured by the HMOs participating in the study, 930 (8.9\%) died during the index admission, amongst the survivors of the index AMI an additional 1265 (13.2\%) died during the first follow-up year and thus were excluded from the study. A flowchart displaying the number of patients as well as inclusion and exclusion for each year is presented in Fig. 1. Baseline characteristics of the patients by study year are presented in Table 1.

Throughout the later study years, patients' age was lower and the rate of male patients was greater compared with earlier years. The prevalence of most traditional cardiovascular risk factors increased (except diabetes mellitus) while the prevalence of most other investigated comorbidities decreased over time. Throughout the later period the rates of STEMI - ST segment elevation myocardial infarction (STEMI) and of 3-vessel or left main coronary artery disease decreased as well. However, the rate of invasive treatments (Percutaneous coronary intervention or/and Coronary artery bypass surgery $[\mathrm{PCI} / \mathrm{CABG}])$ has increased. The rate of adherence with medical therapy during the first year after AMI has improved significantly over time.

\section{Trend of healthcare resources utilization and costs after AMI}

Healthcare resource utilization and costs by study year are displayed in Fig. 2. Throughout the investigated decade the mean length of in-hospital stay and the number of primary care visits decreased significantly (6.65 days 2003 vs. 1.63 days 2015 and 15.6 vs. 11.1 respectively; $p$ for trend $<0.001$ for each). During the same period, the mean number of ED visits, consultant visits and ambulatory visits increased significantly $(0.03$ vs. $0.34, \mathrm{p}$ for trend $<0.001 ; 1.2$ vs. $1.5, \mathrm{p}$ for trend $<0.001 ; 6.2$ vs. 9.0, p for trend $=0.005$ respectively). Mean total costs decreased throughout the investigated period (4579 vs. 3018 USD, $\mathrm{p}$ for trend $<0.001$ ).

\section{Multivariable analysis}

The results of the multivariable models for each investigated outcome are presented in Table 2. The first level models ("unadjusted") show an increased number of ED, ambulatory and consulting clinic visits, while the yearly length of hospital stay, number of primary care visits and the annual total cost decreased during the study period. The second level model, adjusting for time following AMI, shows an increase in the number of ED visits and a decrease in all other parameters. Finally, the third level model which included all variables in addition to patients' baseline characteristics, healthcare service utilization 1 year before AMI and adherence with medical therapy during the first year after AMI, shows a significant decrease in the length of hospital stay, primary care visits and consultant visits, while the yearly number of ED visits has increased and no significant change was observed in the ambulatory visits. No statistically significant difference was found in the adjusted total costs in the third level model.

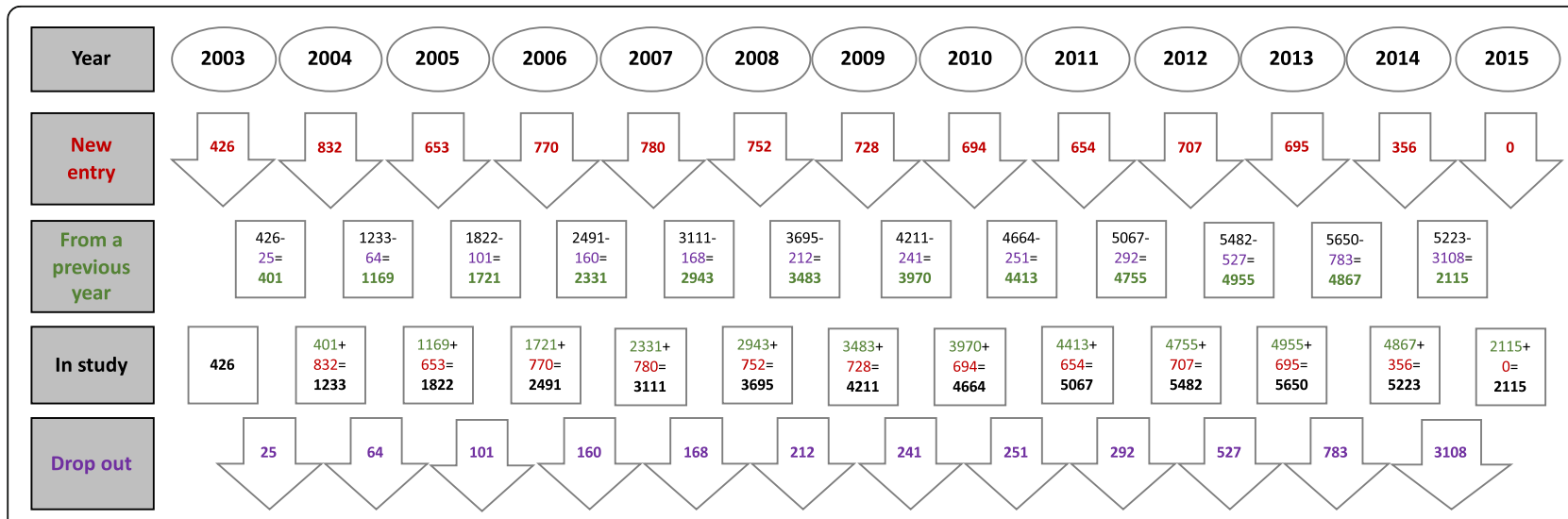

Fig. 1 The study flowchart: number of patients by the study year 
Table 1 The baseline characteristics of the study population by study year

\begin{tabular}{|c|c|c|c|c|c|c|c|c|c|c|c|c|c|c|}
\hline \multirow{2}{*}{$\begin{array}{l}\text { Year } \\
\mathrm{n}\end{array}$} & \multirow{2}{*}{$\begin{array}{l}2003 \\
426\end{array}$} & \multirow{2}{*}{$\begin{array}{l}2004 \\
1233\end{array}$} & \multirow{2}{*}{$\begin{array}{l}2005 \\
1822\end{array}$} & \multirow{2}{*}{$\begin{array}{l}2006 \\
2491\end{array}$} & \multirow{2}{*}{$\begin{array}{l}2007 \\
3111 \\
\end{array}$} & \multirow{2}{*}{$\begin{array}{l}2008 \\
3695 \\
\end{array}$} & \multirow{2}{*}{$\begin{array}{l}2009 \\
4211 \\
\end{array}$} & \multirow{2}{*}{$\begin{array}{l}2010 \\
4664 \\
\end{array}$} & \multirow{2}{*}{$\begin{array}{l}2011 \\
5067 \\
\end{array}$} & \multirow{2}{*}{$\begin{array}{l}2012 \\
5482 \\
\end{array}$} & \multirow{2}{*}{$\begin{array}{l}2013 \\
5650 \\
\end{array}$} & \multirow{2}{*}{$\begin{array}{l}2014 \\
5223 \\
\end{array}$} & \multirow{2}{*}{$\begin{array}{l}2015 \\
2115\end{array}$} & \multirow{2}{*}{$\begin{array}{l}\mathrm{p} \text { for } \\
\text { trend }\end{array}$} \\
\hline & & & & & & & & & & & & & & \\
\hline \multicolumn{15}{|l|}{ Demographics } \\
\hline $\begin{array}{l}\text { Age at AMl, years; } \\
\text { Mean (SD) }\end{array}$ & $\begin{array}{l}64.20 \\
(12.97)\end{array}$ & $\begin{array}{l}64.09 \\
(12.96)\end{array}$ & $\begin{array}{l}63.76 \\
(12.88)\end{array}$ & $\begin{array}{l}63.40 \\
(12.84)\end{array}$ & $\begin{array}{l}63.06 \\
(12.72)\end{array}$ & $\begin{array}{l}63.02 \\
(12.88)\end{array}$ & $\begin{array}{l}62.68 \\
(12.94)\end{array}$ & $\begin{array}{l}62.44 \\
(12.93)\end{array}$ & $\begin{array}{l}62.23 \\
(12.95)\end{array}$ & $\begin{array}{l}62.09 \\
(13.00)\end{array}$ & $\begin{array}{l}61.99 \\
(13.01)\end{array}$ & $\begin{array}{l}61.78 \\
(12.97)\end{array}$ & $\begin{array}{l}60.79 \\
(12.74)\end{array}$ & $<0.001$ \\
\hline Sex, Male & $\begin{array}{l}293 \\
(68.8)\end{array}$ & $\begin{array}{l}850 \\
(68.9)\end{array}$ & $\begin{array}{l}1278 \\
(70.1)\end{array}$ & $\begin{array}{l}1761 \\
(70.7)\end{array}$ & $\begin{array}{l}2235 \\
(71.8)\end{array}$ & $\begin{array}{l}2662 \\
(72.0)\end{array}$ & $\begin{array}{l}3068 \\
(72.9)\end{array}$ & $\begin{array}{l}3404 \\
(73.0)\end{array}$ & $\begin{array}{l}3717 \\
(73.4)\end{array}$ & $\begin{array}{l}4013 \\
(73.2)\end{array}$ & $\begin{array}{l}4165 \\
(73.7)\end{array}$ & $\begin{array}{l}3886 \\
(74.4)\end{array}$ & $\begin{array}{l}1579 \\
(74.7)\end{array}$ & $<0.001$ \\
\hline Muslims & $\begin{array}{l}85 \\
(20.0)\end{array}$ & $\begin{array}{l}246 \\
(20.0)\end{array}$ & $\begin{array}{l}371 \\
(20.4)\end{array}$ & $\begin{array}{l}508 \\
(20.4)\end{array}$ & $\begin{array}{l}642 \\
(20.6)\end{array}$ & $\begin{array}{l}774 \\
(20.9)\end{array}$ & $\begin{array}{l}910 \\
(21.6)\end{array}$ & $\begin{array}{l}1056 \\
(22.6)\end{array}$ & $\begin{array}{l}1180 \\
(23.3)\end{array}$ & $\begin{array}{l}1306 \\
(23.8)\end{array}$ & $\begin{array}{l}1367 \\
(24.2)\end{array}$ & $\begin{array}{l}1310 \\
(25.1)\end{array}$ & $\begin{array}{l}515 \\
(24.3)\end{array}$ & $<0.001$ \\
\hline \multicolumn{15}{|l|}{ Cardiac diseases } \\
\hline $\begin{array}{l}\text { Supraventricular } \\
\text { arrhythmias }\end{array}$ & $\begin{array}{l}46 \\
(10.8)\end{array}$ & $\begin{array}{l}159 \\
(12.9)\end{array}$ & $\begin{array}{l}221 \\
(12.1)\end{array}$ & $\begin{array}{l}306 \\
(12.3)\end{array}$ & $\begin{array}{l}373 \\
(12.0)\end{array}$ & $\begin{array}{l}456 \\
(12.3)\end{array}$ & $\begin{array}{l}497 \\
(11.8)\end{array}$ & $\begin{array}{l}540 \\
(11.6)\end{array}$ & $\begin{array}{l}570 \\
(11.2)\end{array}$ & $\begin{array}{l}616 \\
(11.2)\end{array}$ & $\begin{array}{l}645 \\
(11.4)\end{array}$ & $\begin{array}{l}577 \\
(11.0)\end{array}$ & $\begin{array}{l}223 \\
(10.5)\end{array}$ & 0.003 \\
\hline $\mathrm{CHF}$ & $\begin{array}{l}80 \\
(18.8)\end{array}$ & $\begin{array}{l}186 \\
(15.1)\end{array}$ & $\begin{array}{l}261 \\
(14.3)\end{array}$ & $\begin{array}{l}341 \\
(13.7)\end{array}$ & $\begin{array}{l}390 \\
(12.5)\end{array}$ & $\begin{array}{l}450 \\
(12.2)\end{array}$ & $\begin{array}{l}499 \\
(11.8)\end{array}$ & $\begin{array}{l}525 \\
(11.3)\end{array}$ & $\begin{array}{l}533 \\
(10.5)\end{array}$ & $\begin{array}{l}554 \\
(10.1)\end{array}$ & $\begin{array}{l}555 \\
(9.8)\end{array}$ & $\begin{array}{l}486 \\
(9.3)\end{array}$ & $\begin{array}{l}172 \\
(8.1)\end{array}$ & $<0.001$ \\
\hline $\begin{array}{l}\text { Pulmonary heart } \\
\text { disease }\end{array}$ & $\begin{array}{l}13 \\
(3.1)\end{array}$ & $\begin{array}{l}56 \\
(4.5)\end{array}$ & $\begin{array}{l}88 \\
(4.8)\end{array}$ & $\begin{array}{l}140 \\
(5.6)\end{array}$ & $\begin{array}{l}162 \\
(5.2)\end{array}$ & $\begin{array}{l}214 \\
(5.8)\end{array}$ & $\begin{array}{l}241 \\
(5.7)\end{array}$ & $\begin{array}{l}282 \\
(6.0)\end{array}$ & $\begin{array}{l}307 \\
(6.1)\end{array}$ & $\begin{array}{l}314 \\
(5.7)\end{array}$ & $\begin{array}{l}349 \\
(6.2)\end{array}$ & $\begin{array}{l}308 \\
(5.9)\end{array}$ & $\begin{array}{l}109 \\
(5.2)\end{array}$ & 0.012 \\
\hline $\mathrm{ClHD}$ & $\begin{array}{l}305 \\
(71.6)\end{array}$ & $\begin{array}{l}932 \\
(75.6)\end{array}$ & $\begin{array}{l}1407 \\
(77.2)\end{array}$ & $\begin{array}{l}1928 \\
(77.4)\end{array}$ & $\begin{array}{l}2458 \\
(79.0)\end{array}$ & $\begin{array}{l}2951 \\
(79.9)\end{array}$ & $\begin{array}{l}3426 \\
(81.4)\end{array}$ & $\begin{array}{l}3860 \\
(82.8)\end{array}$ & $\begin{array}{l}4205 \\
(83.0)\end{array}$ & $\begin{array}{l}4565 \\
(83.3)\end{array}$ & $\begin{array}{l}4761 \\
(84.3)\end{array}$ & $\begin{array}{l}4447 \\
(85.1)\end{array}$ & $\begin{array}{l}1820 \\
(86.1)\end{array}$ & $<0.001$ \\
\hline AV block & $\begin{array}{l}14 \\
(3.3)\end{array}$ & $\begin{array}{l}42 \\
(3.4)\end{array}$ & $\begin{array}{l}68 \\
(3.7)\end{array}$ & $\begin{array}{l}92 \\
(3.7)\end{array}$ & $\begin{array}{l}111 \\
(3.6)\end{array}$ & $\begin{array}{l}118 \\
(3.2)\end{array}$ & $\begin{array}{l}129 \\
(3.1)\end{array}$ & $\begin{array}{l}148 \\
(3.2)\end{array}$ & $\begin{array}{l}159 \\
(3.1)\end{array}$ & $\begin{array}{l}177 \\
(3.2)\end{array}$ & $\begin{array}{l}183 \\
(3.2)\end{array}$ & $\begin{array}{l}161 \\
(3.1)\end{array}$ & $\begin{array}{l}63 \\
(3.0)\end{array}$ & 0.092 \\
\hline \multicolumn{15}{|l|}{ Cardiovascular risk factors } \\
\hline Renal diseases & $\begin{array}{l}206 \\
(48.4)\end{array}$ & $\begin{array}{l}479 \\
(38.8)\end{array}$ & $\begin{array}{l}640 \\
(35.1)\end{array}$ & $\begin{array}{l}844 \\
(33.9)\end{array}$ & $\begin{array}{l}1001 \\
(32.2)\end{array}$ & $\begin{array}{l}1125 \\
(30.4)\end{array}$ & $\begin{array}{l}1244 \\
(29.5)\end{array}$ & $\begin{array}{l}1335 \\
(28.6)\end{array}$ & $\begin{array}{l}1416 \\
(27.9)\end{array}$ & $\begin{array}{l}1499 \\
(27.3)\end{array}$ & $\begin{array}{l}1447 \\
(25.6)\end{array}$ & $\begin{array}{l}1296 \\
(24.8)\end{array}$ & $\begin{array}{l}504 \\
(23.8)\end{array}$ & $<0.001$ \\
\hline Diabetes Mellitus & $\begin{array}{l}157 \\
(36.9)\end{array}$ & $\begin{array}{l}409 \\
(33.2)\end{array}$ & $\begin{array}{l}598 \\
(32.8)\end{array}$ & $\begin{array}{l}801 \\
(32.2)\end{array}$ & $\begin{array}{l}1028 \\
(33.0)\end{array}$ & $\begin{array}{l}1250 \\
(33.8)\end{array}$ & $\begin{array}{l}1416 \\
(33.6)\end{array}$ & $\begin{array}{l}1536 \\
(32.9)\end{array}$ & $\begin{array}{l}1678 \\
(33.1)\end{array}$ & $\begin{array}{l}1802 \\
(32.9)\end{array}$ & $\begin{array}{l}1908 \\
(33.8)\end{array}$ & $\begin{array}{l}1770 \\
(33.9)\end{array}$ & $\begin{array}{l}681 \\
(32.2)\end{array}$ & 0.825 \\
\hline Dyslipidemia & $\begin{array}{l}291 \\
(68.3)\end{array}$ & $\begin{array}{l}815 \\
(66.1)\end{array}$ & $\begin{array}{l}1236 \\
(67.8)\end{array}$ & $\begin{array}{l}1671 \\
(67.1)\end{array}$ & $\begin{array}{l}2140 \\
(68.8)\end{array}$ & $\begin{array}{l}2555 \\
(69.1)\end{array}$ & $\begin{array}{l}2969 \\
(70.5)\end{array}$ & $\begin{array}{l}3331 \\
(71.4)\end{array}$ & $\begin{array}{l}3680 \\
(72.6)\end{array}$ & $\begin{array}{l}4006 \\
(73.1)\end{array}$ & $\begin{array}{l}4170 \\
(73.8)\end{array}$ & $\begin{array}{l}3871 \\
(74.1)\end{array}$ & $\begin{array}{l}1563 \\
(73.9)\end{array}$ & $<0.001$ \\
\hline Hypertension & $\begin{array}{l}200 \\
(46.9)\end{array}$ & $\begin{array}{l}648 \\
(52.6)\end{array}$ & $\begin{array}{l}929 \\
(51.0)\end{array}$ & $\begin{array}{l}1252 \\
(50.3)\end{array}$ & $\begin{array}{l}1556 \\
(50.0)\end{array}$ & $\begin{array}{l}1872 \\
(50.7)\end{array}$ & $\begin{array}{l}2114 \\
(50.2)\end{array}$ & $\begin{array}{l}2368 \\
(50.8)\end{array}$ & $\begin{array}{l}2575 \\
(50.8)\end{array}$ & $\begin{array}{l}2796 \\
(51.0)\end{array}$ & $\begin{array}{l}2921 \\
(51.7)\end{array}$ & $\begin{array}{l}2670 \\
(51.1)\end{array}$ & $\begin{array}{l}1074 \\
(50.8)\end{array}$ & 0.253 \\
\hline Obesity & $\begin{array}{l}87 \\
(20.4)\end{array}$ & $\begin{array}{l}255 \\
(20.7)\end{array}$ & $\begin{array}{l}369 \\
(20.3)\end{array}$ & $\begin{array}{l}535 \\
(21.5)\end{array}$ & $\begin{array}{l}683 \\
(22.0)\end{array}$ & $\begin{array}{l}849 \\
(23.0)\end{array}$ & $\begin{array}{l}1001 \\
(23.8)\end{array}$ & $\begin{array}{l}1145 \\
(24.5)\end{array}$ & $\begin{array}{l}1254 \\
(24.7)\end{array}$ & $\begin{array}{l}1368 \\
(25.0)\end{array}$ & $\begin{array}{l}1414 \\
(25.0)\end{array}$ & $\begin{array}{l}1302 \\
(24.9)\end{array}$ & $\begin{array}{l}553 \\
(26.1)\end{array}$ & $<0.001$ \\
\hline Smoking & $\begin{array}{l}168 \\
(39.4)\end{array}$ & $\begin{array}{l}487 \\
(39.5)\end{array}$ & $\begin{array}{l}770 \\
(42.3)\end{array}$ & $\begin{array}{l}1064 \\
(42.7)\end{array}$ & $\begin{array}{l}1359 \\
(43.7)\end{array}$ & $\begin{array}{l}1617 \\
(43.8)\end{array}$ & $\begin{array}{l}1896 \\
(45.0)\end{array}$ & $\begin{array}{l}2110 \\
(45.2)\end{array}$ & $\begin{array}{l}2313 \\
(45.6)\end{array}$ & $\begin{array}{l}2524 \\
(46.0)\end{array}$ & $\begin{array}{l}2616 \\
(46.3)\end{array}$ & $\begin{array}{l}2451 \\
(46.9)\end{array}$ & $\begin{array}{l}1030 \\
(48.7)\end{array}$ & $<0.001$ \\
\hline PVD & $\begin{array}{l}58 \\
(13.6)\end{array}$ & $\begin{array}{l}165 \\
(13.4)\end{array}$ & $\begin{array}{l}245 \\
(13.4)\end{array}$ & $\begin{array}{l}305 \\
(12.2)\end{array}$ & $\begin{array}{l}365 \\
(11.7)\end{array}$ & $\begin{array}{l}411 \\
(11.1)\end{array}$ & $\begin{array}{l}444 \\
(10.5)\end{array}$ & $\begin{array}{l}471 \\
(10.1)\end{array}$ & $\begin{array}{l}492 \\
(9.7)\end{array}$ & $\begin{array}{l}501 \\
(9.1)\end{array}$ & $\begin{array}{l}472 \\
(8.4)\end{array}$ & $\begin{array}{l}416 \\
(8.0)\end{array}$ & $\begin{array}{l}164 \\
(7.8)\end{array}$ & $<0.001$ \\
\hline Family history of IHD & $\begin{array}{l}18 \\
(4.2)\end{array}$ & $\begin{array}{l}60 \\
(4.9)\end{array}$ & $\begin{array}{l}109 \\
(6.0)\end{array}$ & $\begin{array}{l}169 \\
(6.8)\end{array}$ & $\begin{array}{l}225 \\
(7.2)\end{array}$ & $\begin{array}{l}292 \\
(7.9)\end{array}$ & $\begin{array}{l}351 \\
(8.3)\end{array}$ & $\begin{array}{l}419 \\
(9.0)\end{array}$ & $\begin{array}{l}469 \\
(9.3)\end{array}$ & $\begin{array}{l}512 \\
(9.3)\end{array}$ & $\begin{array}{l}543 \\
(9.6)\end{array}$ & $\begin{array}{l}531 \\
(10.2)\end{array}$ & $\begin{array}{l}237 \\
(11.2)\end{array}$ & $<0.001$ \\
\hline \multicolumn{15}{|l|}{ Other disorders } \\
\hline COPD & $\begin{array}{l}20 \\
(4.7)\end{array}$ & $\begin{array}{l}79 \\
(6.4)\end{array}$ & $\begin{array}{l}121 \\
(6.6)\end{array}$ & $\begin{array}{l}155 \\
(6.2)\end{array}$ & $\begin{array}{l}182 \\
(5.9)\end{array}$ & $\begin{array}{l}206 \\
(5.6)\end{array}$ & $\begin{array}{l}220 \\
(5.2)\end{array}$ & $\begin{array}{l}239 \\
(5.1)\end{array}$ & $\begin{array}{l}247 \\
(4.9)\end{array}$ & $\begin{array}{l}261 \\
(4.8)\end{array}$ & $\begin{array}{l}273 \\
(4.8)\end{array}$ & $\begin{array}{l}235 \\
(4.5)\end{array}$ & $\begin{array}{l}87 \\
(4.1)\end{array}$ & $<0.001$ \\
\hline $\begin{array}{l}\text { Neurological } \\
\text { disorders }\end{array}$ & $\begin{array}{l}47 \\
(11.0)\end{array}$ & $\begin{array}{l}139 \\
(11.3)\end{array}$ & $\begin{array}{l}207 \\
(11.4)\end{array}$ & $\begin{array}{l}277 \\
(11.1)\end{array}$ & $\begin{array}{l}321 \\
(10.3)\end{array}$ & $\begin{array}{l}399 \\
(10.8)\end{array}$ & $\begin{array}{l}465 \\
(11.0)\end{array}$ & $\begin{array}{l}508 \\
(10.9)\end{array}$ & $\begin{array}{l}570 \\
(11.2)\end{array}$ & $\begin{array}{l}623 \\
(11.4)\end{array}$ & $\begin{array}{l}640 \\
(11.3)\end{array}$ & $\begin{array}{l}563 \\
(10.8)\end{array}$ & $\begin{array}{l}210 \\
(9.9)\end{array}$ & 0.841 \\
\hline Anemia & $\begin{array}{l}199 \\
(46.7)\end{array}$ & $\begin{array}{l}576 \\
(46.7)\end{array}$ & $\begin{array}{l}836 \\
(45.9)\end{array}$ & $\begin{array}{l}1140 \\
(45.8)\end{array}$ & $\begin{array}{l}1414 \\
(45.5)\end{array}$ & $\begin{array}{l}1737 \\
(47.0)\end{array}$ & $\begin{array}{l}1975 \\
(46.9)\end{array}$ & $\begin{array}{l}2215 \\
(47.5)\end{array}$ & $\begin{array}{l}2409 \\
(47.5)\end{array}$ & $\begin{array}{l}2578 \\
(47.0)\end{array}$ & $\begin{array}{l}2670 \\
(47.3)\end{array}$ & $\begin{array}{l}2440 \\
(46.7)\end{array}$ & $\begin{array}{l}944 \\
(44.6)\end{array}$ & 0.530 \\
\hline Gl bleeding & $6(1.4)$ & $\begin{array}{l}20 \\
(1.6)\end{array}$ & $\begin{array}{l}35 \\
(1.9)\end{array}$ & $\begin{array}{l}48 \\
(1.9)\end{array}$ & $\begin{array}{l}60 \\
(1.9)\end{array}$ & $\begin{array}{l}61 \\
(1.7)\end{array}$ & $\begin{array}{l}73 \\
(1.7)\end{array}$ & $\begin{array}{l}78 \\
(1.7)\end{array}$ & $\begin{array}{l}84 \\
(1.7)\end{array}$ & $\begin{array}{l}94 \\
(1.7)\end{array}$ & $\begin{array}{l}97 \\
(1.7)\end{array}$ & $\begin{array}{l}84 \\
(1.6)\end{array}$ & $\begin{array}{l}35 \\
(1.7)\end{array}$ & 0.391 \\
\hline $\begin{array}{l}\text { Schizophrenia/ } \\
\text { Psychosis }\end{array}$ & $5(1.2)$ & $\begin{array}{l}18 \\
(1.5)\end{array}$ & $\begin{array}{l}22 \\
(1.2)\end{array}$ & $\begin{array}{l}40 \\
(1.6)\end{array}$ & $\begin{array}{l}50 \\
(1.6)\end{array}$ & $\begin{array}{l}59 \\
(1.6)\end{array}$ & $\begin{array}{l}59 \\
(1.4)\end{array}$ & $\begin{array}{l}62 \\
(1.3)\end{array}$ & $\begin{array}{l}63 \\
(1.2)\end{array}$ & $\begin{array}{l}62 \\
(1.1)\end{array}$ & $\begin{array}{l}65 \\
(1.2)\end{array}$ & $\begin{array}{l}54 \\
(1.0)\end{array}$ & $\begin{array}{l}17 \\
(0.8)\end{array}$ & 0.001 \\
\hline $\begin{array}{l}\text { Alcohol/drug } \\
\text { addiction }\end{array}$ & $\begin{array}{l}9 \\
(2.1)\end{array}$ & $\begin{array}{l}20 \\
(1.6)\end{array}$ & $\begin{array}{l}30 \\
(1.6)\end{array}$ & $\begin{array}{l}48 \\
(1.9)\end{array}$ & $\begin{array}{l}67 \\
(2.2)\end{array}$ & $\begin{array}{l}76 \\
(2.1)\end{array}$ & $\begin{array}{l}79 \\
(1.9)\end{array}$ & $\begin{array}{l}90 \\
(1.9)\end{array}$ & $\begin{array}{l}96 \\
(1.9)\end{array}$ & $\begin{array}{l}106 \\
(1.9)\end{array}$ & $\begin{array}{l}105 \\
(1.9)\end{array}$ & $\begin{array}{l}97 \\
(1.9)\end{array}$ & $\begin{array}{l}37 \\
(1.7)\end{array}$ & 0.743 \\
\hline Malignancy & $\begin{array}{l}8 \\
(1.9)\end{array}$ & $\begin{array}{l}34 \\
(2.8)\end{array}$ & $\begin{array}{l}45 \\
(2.5)\end{array}$ & $\begin{array}{l}56 \\
(2.2)\end{array}$ & $\begin{array}{l}69 \\
(2.2)\end{array}$ & $\begin{array}{l}87 \\
(2.4)\end{array}$ & $\begin{array}{l}97 \\
(2.3)\end{array}$ & $\begin{array}{l}98 \\
(2.1)\end{array}$ & $\begin{array}{l}106 \\
(2.1)\end{array}$ & $\begin{array}{l}119 \\
(2.2)\end{array}$ & $\begin{array}{l}128 \\
(2.3)\end{array}$ & $\begin{array}{l}121 \\
(2.3)\end{array}$ & $\begin{array}{l}43 \\
(2.0)\end{array}$ & 0.430 \\
\hline
\end{tabular}


Table 1 The baseline characteristics of the study population by study year (Continued)

\begin{tabular}{|c|c|c|c|c|c|c|c|c|c|c|c|c|c|c|}
\hline Year & 2003 & 2004 & 2005 & 2006 & 2007 & 2008 & 2009 & 2010 & 2011 & 2012 & 2013 & 2014 & 2015 & for \\
\hline$n$ & 426 & 1233 & 1822 & 2491 & 3111 & 3695 & 4211 & 4664 & 5067 & 5482 & 5650 & 5223 & 2115 & \\
\hline $\begin{array}{l}\text { Admitted/transposed } \\
\text { to ICCU }\end{array}$ & $\begin{array}{l}288 \\
(67.6)\end{array}$ & $\begin{array}{l}869 \\
(70.5)\end{array}$ & $\begin{array}{l}1339 \\
(73.5)\end{array}$ & $\begin{array}{l}1848 \\
(74.2)\end{array}$ & $\begin{array}{l}2352 \\
(75.6)\end{array}$ & $\begin{array}{l}2797 \\
(75.7)\end{array}$ & $\begin{array}{l}3233 \\
(76.8)\end{array}$ & $\begin{array}{l}3635 \\
(77.9)\end{array}$ & $\begin{array}{l}3963 \\
(78.2)\end{array}$ & $\begin{array}{l}4264 \\
(77.8)\end{array}$ & $\begin{array}{l}4401 \\
(77.9)\end{array}$ & $\begin{array}{l}4101 \\
(78.5)\end{array}$ & $\begin{array}{l}1707 \\
(80.7)\end{array}$ & $<0.001$ \\
\hline $\begin{array}{l}\text { Length of hospital stay } \\
\text { (days), }>7\end{array}$ & $\begin{array}{l}232 \\
(54.5)\end{array}$ & $\begin{array}{l}666 \\
(54.0)\end{array}$ & $\begin{array}{l}986 \\
(54.1)\end{array}$ & $\begin{array}{l}1375 \\
(55.2)\end{array}$ & $\begin{array}{l}1668 \\
(53.6)\end{array}$ & $\begin{array}{l}1924 \\
(52.1)\end{array}$ & $\begin{array}{l}2127 \\
(50.5)\end{array}$ & $\begin{array}{l}2308 \\
(49.5)\end{array}$ & $\begin{array}{l}2439 \\
(48.1)\end{array}$ & $\begin{array}{l}2594 \\
(47.3)\end{array}$ & $\begin{array}{l}2599 \\
(46.0)\end{array}$ & $\begin{array}{l}2339 \\
(44.8)\end{array}$ & $\begin{array}{l}909 \\
(43.0)\end{array}$ & $<0.00$ \\
\hline Type of AMI, STEMI & $\begin{array}{l}323 \\
(75.8)\end{array}$ & $\begin{array}{l}816 \\
(66.2)\end{array}$ & $\begin{array}{l}1188 \\
(65.2)\end{array}$ & $\begin{array}{l}1531 \\
(61.5)\end{array}$ & $\begin{array}{l}1826 \\
(58.7)\end{array}$ & $\begin{array}{l}2087 \\
(56.5)\end{array}$ & $\begin{array}{l}2331 \\
(55.4)\end{array}$ & $\begin{array}{l}2545 \\
(54.6)\end{array}$ & $\begin{array}{l}2750 \\
(54.3)\end{array}$ & $\begin{array}{l}2961 \\
(54.0)\end{array}$ & $\begin{array}{l}2966 \\
(52.5)\end{array}$ & $\begin{array}{l}2722 \\
(52.1)\end{array}$ & $\begin{array}{l}1130 \\
(53.4)\end{array}$ & 0.00 \\
\hline \multicolumn{15}{|l|}{ Results of echocardiography } \\
\hline $\begin{array}{l}\text { Echocardiography } \\
\text { performance }\end{array}$ & $\begin{array}{l}328 \\
(77.0)\end{array}$ & $\begin{array}{l}974 \\
(79.0)\end{array}$ & $\begin{array}{l}1482 \\
(81.3)\end{array}$ & $\begin{array}{l}2054 \\
(82.5)\end{array}$ & $\begin{array}{l}2620 \\
(84.2)\end{array}$ & $\begin{array}{l}3117 \\
(84.4)\end{array}$ & $\begin{array}{l}3606 \\
(85.6)\end{array}$ & $\begin{array}{l}4043 \\
(86.7)\end{array}$ & $\begin{array}{l}4421 \\
(87.3)\end{array}$ & $\begin{array}{l}4784 \\
(87.3)\end{array}$ & $\begin{array}{l}4945 \\
(87.5)\end{array}$ & $\begin{array}{l}4624 \\
(88.5)\end{array}$ & $\begin{array}{l}1904 \\
(90.0)\end{array}$ & $<0.00$ \\
\hline $\begin{array}{l}\text { Sever } \\
\text { dysfu }\end{array}$ & $\begin{array}{l}31 \\
(9.5)\end{array}$ & $\begin{array}{l}91 \\
(9.3)\end{array}$ & $\begin{array}{l}135 \\
(9.1)\end{array}$ & $\begin{array}{l}176 \\
(8.6)\end{array}$ & $\begin{array}{l}210 \\
(8.0)\end{array}$ & $\begin{array}{l}234 \\
(7.5)\end{array}$ & $\begin{array}{l}244 \\
(6.8)\end{array}$ & $\begin{array}{l}264 \\
(6.5)\end{array}$ & $\begin{array}{l}291 \\
(6.6)\end{array}$ & $\begin{array}{l}332 \\
(6.9)\end{array}$ & $\begin{array}{l}358 \\
(7.2)\end{array}$ & $\begin{array}{l}346 \\
(7.5)\end{array}$ & $\begin{array}{l}117 \\
(6.1)\end{array}$ & $<0.001$ \\
\hline LV hypertrophy & $\begin{array}{l}24 \\
(7.3)\end{array}$ & $\begin{array}{l}52 \\
(5.3)\end{array}$ & $\begin{array}{l}67 \\
(4.5)\end{array}$ & $\begin{array}{l}84 \\
(4.1)\end{array}$ & $\begin{array}{l}102 \\
(3.9)\end{array}$ & $\begin{array}{l}125 \\
(4.0)\end{array}$ & $\begin{array}{l}143 \\
(4.0)\end{array}$ & $\begin{array}{l}150 \\
(3.7)\end{array}$ & $\begin{array}{l}143 \\
(3.2)\end{array}$ & $\begin{array}{l}163 \\
(3.4)\end{array}$ & $\begin{array}{l}162 \\
(3.3)\end{array}$ & & $\begin{array}{l}65 \\
(3.4)\end{array}$ & $<0.00$ \\
\hline Mitral regurgitation & $\begin{array}{l}13 \\
(4.0)\end{array}$ & $\begin{array}{l}37 \\
(3.8)\end{array}$ & $\begin{array}{l}61 \\
(4.1)\end{array}$ & $\begin{array}{l}82 \\
(4.0)\end{array}$ & $\begin{array}{l}100 \\
(3.8)\end{array}$ & $\begin{array}{l}113 \\
(3.6)\end{array}$ & $\begin{array}{l}144 \\
(4.0)\end{array}$ & $\begin{array}{l}169 \\
(4.2)\end{array}$ & $\begin{array}{l}200 \\
(4.5)\end{array}$ & & & & $\begin{array}{l}61 \\
(3.2)\end{array}$ & 0.717 \\
\hline $\begin{array}{l}\text { Tricuspidal } \\
\text { regurgitation }\end{array}$ & $\begin{array}{l}8 \\
(2.4)\end{array}$ & $\begin{array}{l}19 \\
(2.0)\end{array}$ & $\begin{array}{l}31 \\
(2.1)\end{array}$ & $\begin{array}{l}44 \\
(2.1)\end{array}$ & $\begin{array}{l}53 \\
(2.0)\end{array}$ & $\begin{array}{l}73 \\
(2.3)\end{array}$ & $\begin{array}{l}80 \\
(2.2)\end{array}$ & $\begin{array}{l}90 \\
(2.2)\end{array}$ & $\begin{array}{l}100 \\
(2.3)\end{array}$ & & & & $\begin{array}{l}39 \\
(2.0)\end{array}$ & 0.571 \\
\hline ulmonary hypertension & $\begin{array}{l}13 \\
(4.0)\end{array}$ & $\begin{array}{l}37 \\
(3.8)\end{array}$ & $\begin{array}{l}59 \\
(4.0)\end{array}$ & $\begin{array}{l}91 \\
(4.4)\end{array}$ & $\begin{array}{l}119 \\
(4.5)\end{array}$ & $\begin{array}{l}159 \\
(5.1)\end{array}$ & $\begin{array}{l}182 \\
(5.0)\end{array}$ & $\begin{array}{l}211 \\
(5.2)\end{array}$ & $\begin{array}{l}225 \\
(5.1)\end{array}$ & $\begin{array}{l}234 \\
(4.9)\end{array}$ & & $\begin{array}{l}211 \\
(4.6)\end{array}$ & $\begin{array}{l}78 \\
(4.1)\end{array}$ & 0.418 \\
\hline \multicolumn{15}{|l|}{ Results of angiography } \\
\hline $\begin{array}{l}\text { Angiogr } \\
\text { perform }\end{array}$ & $\begin{array}{l}238 \\
(55.9)\end{array}$ & $\begin{array}{l}795 \\
(64.5)\end{array}$ & $\begin{array}{l}1260 \\
(69.2)\end{array}$ & $\begin{array}{l}1731 \\
(69.5)\end{array}$ & $\begin{array}{l}2250 \\
(72.3)\end{array}$ & $\begin{array}{l}2749 \\
(74.4)\end{array}$ & $\begin{array}{l}3225 \\
(76.6)\end{array}$ & $\begin{array}{l}3666 \\
(78.6)\end{array}$ & $\begin{array}{l}4039 \\
(79.7)\end{array}$ & $\begin{array}{l}4418 \\
(80.6)\end{array}$ & $\begin{array}{l}4646 \\
(82.2)\end{array}$ & $\begin{array}{l}4362 \\
(83.5)\end{array}$ & $\begin{array}{l}1774 \\
(83.9)\end{array}$ & 0.00 \\
\hline $\begin{array}{l}\text { Measure of CAD, No } \\
\text { or non-significant }\end{array}$ & $\begin{array}{l}7 \\
(2.9)\end{array}$ & $\begin{array}{l}42 \\
(5.3)\end{array}$ & $\begin{array}{l}62 \\
(4.9)\end{array}$ & $\begin{array}{l}85 \\
(4.9)\end{array}$ & $\begin{array}{l}105 \\
(4.7)\end{array}$ & $\begin{array}{l}125 \\
(4.5)\end{array}$ & $\begin{array}{l}150 \\
(4.7)\end{array}$ & $\begin{array}{l}173 \\
(4.7)\end{array}$ & $\begin{array}{l}196 \\
(4.9)\end{array}$ & & $\begin{array}{l}240 \\
(5.2)\end{array}$ & $\begin{array}{l}216 \\
(5.0)\end{array}$ & $\begin{array}{l}75 \\
(4.2)\end{array}$ & 0.001 \\
\hline One vessel & $\begin{array}{l}50 \\
(21.0)\end{array}$ & $\begin{array}{l}177 \\
(22.3)\end{array}$ & $\begin{array}{l}279 \\
(22.1)\end{array}$ & $\begin{array}{l}412 \\
(23.8)\end{array}$ & $\begin{array}{l}554 \\
(24.6)\end{array}$ & $\begin{array}{l}705 \\
(25.6)\end{array}$ & $\begin{array}{l}832 \\
(25.8)\end{array}$ & $\begin{array}{l}958 \\
(26.1)\end{array}$ & $\begin{array}{l}1083 \\
(26.8)\end{array}$ & $\begin{array}{l}1205 \\
(27.3)\end{array}$ & $\begin{array}{l}1277 \\
(27.5)\end{array}$ & $\begin{array}{l}1236 \\
(28.3)\end{array}$ & $\begin{array}{l}534 \\
(30.1)\end{array}$ & $\begin{array}{l}<0.001 \\
<0.001\end{array}$ \\
\hline Two vessels & $\begin{array}{l}71 \\
(29.8)\end{array}$ & $\begin{array}{l}221 \\
(27.8)\end{array}$ & $\begin{array}{l}358 \\
(28.4)\end{array}$ & $\begin{array}{l}496 \\
(28.7)\end{array}$ & $\begin{array}{l}653 \\
(29.0)\end{array}$ & $\begin{array}{l}788 \\
(28.7)\end{array}$ & $\begin{array}{l}902 \\
(28.0)\end{array}$ & $\begin{array}{l}1041 \\
(28.4)\end{array}$ & $\begin{array}{l}1143 \\
(28.3)\end{array}$ & $\begin{array}{l}1255 \\
(28.4)\end{array}$ & $\begin{array}{l}1325 \\
(28.5)\end{array}$ & $\begin{array}{l}1262 \\
(28.9)\end{array}$ & $\begin{array}{l}506 \\
(28.5)\end{array}$ & \\
\hline Three vessels/ LM & $\begin{array}{l}110 \\
(46.2)\end{array}$ & $\begin{array}{l}355 \\
(44.7)\end{array}$ & $\begin{array}{l}561 \\
(44.5)\end{array}$ & $\begin{array}{l}738 \\
(42.6)\end{array}$ & $\begin{array}{l}938 \\
(41.7)\end{array}$ & $\begin{array}{l}1130 \\
(41.1)\end{array}$ & $\begin{array}{l}1340 \\
(41.6)\end{array}$ & $\begin{array}{l}1494 \\
(40.8)\end{array}$ & $\begin{array}{l}1617 \\
(40.0)\end{array}$ & $\begin{array}{l}1743 \\
(39.5)\end{array}$ & $\begin{array}{l}1804 \\
(38.8)\end{array}$ & $\begin{array}{l}1648 \\
(37.8)\end{array}$ & $\begin{array}{l}659 \\
(37.1)\end{array}$ & \\
\hline $\begin{array}{l}\text { Type of treatment, } \\
\text { Noninvasive }\end{array}$ & $\begin{array}{l}179 \\
(42.0)\end{array}$ & $\begin{array}{l}418 \\
(33.9)\end{array}$ & $\begin{array}{l}533 \\
(29.3)\end{array}$ & $\begin{array}{l}687 \\
(27.6)\end{array}$ & $\begin{array}{l}770 \\
(24.8)\end{array}$ & $\begin{array}{l}841 \\
(22.8)\end{array}$ & $\begin{array}{l}868 \\
(20.6)\end{array}$ & $\begin{array}{l}862 \\
(18.5)\end{array}$ & $\begin{array}{l}879 \\
(17.3)\end{array}$ & $\begin{array}{l}899 \\
(16.4)\end{array}$ & $\begin{array}{l}833 \\
(14.7)\end{array}$ & $\begin{array}{l}691 \\
(13.2)\end{array}$ & $\begin{array}{l}258 \\
(12.2)\end{array}$ & \\
\hline $\mathrm{PCl}$ & $\begin{array}{l}203 \\
(47.7)\end{array}$ & $\begin{array}{l}673 \\
(54.6)\end{array}$ & $\begin{array}{l}1070 \\
(58.7)\end{array}$ & $\begin{array}{l}1506 \\
(60.5)\end{array}$ & $\begin{array}{l}1960 \\
(63.0)\end{array}$ & $\begin{array}{l}2360 \\
(63.9)\end{array}$ & $\begin{array}{l}2725 \\
(64.7)\end{array}$ & $\begin{array}{l}3079 \\
(66.0)\end{array}$ & $\begin{array}{l}3391 \\
(66.9)\end{array}$ & $\begin{array}{l}3707 \\
(67.6)\end{array}$ & $\begin{array}{l}3908 \\
(69.2)\end{array}$ & $\begin{array}{l}3669 \\
(70.2)\end{array}$ & $\begin{array}{l}1512 \\
(71.5)\end{array}$ & $<0.00$ \\
\hline CABG & $\begin{array}{l}44 \\
(10.3)\end{array}$ & $\begin{array}{l}142 \\
(11.5)\end{array}$ & $\begin{array}{l}219 \\
(12.0)\end{array}$ & $\begin{array}{l}298 \\
(12.0)\end{array}$ & $\begin{array}{l}381 \\
(12.2)\end{array}$ & $\begin{array}{l}494 \\
(13.4)\end{array}$ & $\begin{array}{l}618 \\
(14.7)\end{array}$ & $\begin{array}{l}723 \\
(15.5)\end{array}$ & $\begin{array}{l}797 \\
(15.7)\end{array}$ & $\begin{array}{l}875 \\
(16.0)\end{array}$ & $\begin{array}{l}908 \\
(16.1)\end{array}$ & $\begin{array}{l}862 \\
(16.5)\end{array}$ & $\begin{array}{l}345 \\
(16.3)\end{array}$ & \\
\hline
\end{tabular}

Healthcare utilization an year before AMl; Mean (SD)

\begin{tabular}{|c|c|c|c|c|c|c|c|c|c|c|c|c|c|c|}
\hline $\begin{array}{l}\text { Hospitalizations } \\
\text { LOS, days }\end{array}$ & $\begin{array}{l}4.28 \\
(7.74)\end{array}$ & $\begin{array}{l}4.61 \\
(9.79)\end{array}$ & $\begin{array}{l}4.47 \\
(10.45)\end{array}$ & $\begin{array}{l}4.38 \\
(9.81)\end{array}$ & $\begin{array}{l}4.1 \\
(8.49)\end{array}$ & $\begin{array}{l}4.04 \\
(8.47)\end{array}$ & $\begin{array}{l}3.73 \\
(7.79)\end{array}$ & $\begin{array}{l}3.59 \\
(7.49)\end{array}$ & $\begin{array}{l}3.49 \\
(8.34)\end{array}$ & $\begin{array}{l}3.27 \\
(8.02)\end{array}$ & $\begin{array}{l}3.03 \\
(7.90)\end{array}$ & $\begin{array}{l}2.8 \\
(7.76)\end{array}$ & $\begin{array}{l}2.82 \\
(8.27)\end{array}$ & 0.001 \\
\hline ED visits & $(0.17)$ & $(0.20)$ & $(0.20)$ & $\begin{array}{l}0.03 \\
(0.19)\end{array}$ & tat & 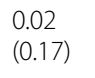 & (0) & $(0$. & $(0$ & & & & & 0.001 \\
\hline $\begin{array}{l}\text { Number of primary } \\
\text { clinic visits }\end{array}$ & & & & & & & & & & & $\begin{array}{l}10.72 \\
(9.97)\end{array}$ & & & .001 \\
\hline ibulatory & $(8.52)$ & (10.64) & ( & $(10$ & o0) & 9) & $0)$ & 7) & $\begin{array}{l}6.86 \\
(14.96)\end{array}$ & & $\begin{array}{l}6.64 \\
(13.27)\end{array}$ & 8) & 45) & .001 \\
\hline $\begin{array}{l}\text { Number of consulting } \\
\text { clinic visits }\end{array}$ & $\begin{array}{l}0.53 \\
(1.25)\end{array}$ & $\begin{array}{l}0.78 \\
(1.68)\end{array}$ & $\begin{array}{l}0.82 \\
(1.70)\end{array}$ & $\begin{array}{l}0.93 \\
(1.85)\end{array}$ & $\begin{array}{l}0.94 \\
(1.83)\end{array}$ & & $\begin{array}{l}0.98 \\
(1.83)\end{array}$ & $\begin{array}{l}1.02 \\
(1.92)\end{array}$ & $\begin{array}{l}1.03 \\
(1.92)\end{array}$ & & $\begin{array}{l}1.06 \\
(1.96)\end{array}$ & & & $<0.001$ \\
\hline Total cos & $\begin{array}{l}2391 \\
(4029)\end{array}$ & $\begin{array}{l}2962 \\
(5066)\end{array}$ & $\begin{array}{l}2909 \\
(4878)\end{array}$ & $\begin{array}{l}3055 \\
(5516)\end{array}$ & $\begin{array}{l}3090 \\
(5457)\end{array}$ & $\begin{array}{l}3136 \\
(5458)\end{array}$ & $\begin{array}{l}3043 \\
(4819)\end{array}$ & $\begin{array}{l}3109 \\
(5231)\end{array}$ & $\begin{array}{l}3208 \\
(6453)\end{array}$ & $\begin{array}{l}3124 \\
(6182)\end{array}$ & $\begin{array}{l}3006 \\
(5675)\end{array}$ & $\begin{array}{l}2922 \\
(5585)\end{array}$ & $\begin{array}{l}2966 \\
(6618)\end{array}$ & 0.996 \\
\hline \multicolumn{15}{|l|}{ ow-up } \\
\hline medical treatment & $\begin{array}{l}64 \\
(15.0)\end{array}$ & $\begin{array}{l}218 \\
(17.7)\end{array}$ & $\begin{array}{l}362 \\
(19.9)\end{array}$ & $\begin{array}{l}553 \\
(22.2)\end{array}$ & $\begin{array}{l}724 \\
(23.3)\end{array}$ & (24.0) & (25.3) & $\begin{array}{l}1204 \\
(25.8)\end{array}$ & $(25.5)$ & (24.7) & $\begin{array}{l}1378 \\
(24.4)\end{array}$ & $\begin{array}{l}1275 \\
(24.4)\end{array}$ & $\begin{array}{l}529 \\
(25.0)\end{array}$ & .001 \\
\hline
\end{tabular}


Table 1 The baseline characteristics of the study population by study year (Continued)

\begin{tabular}{|c|c|c|c|c|c|c|c|c|c|c|c|c|c|c|}
\hline Year & 2003 & 2004 & 2005 & 2006 & 2007 & 2008 & 2009 & 2010 & 2011 & 2012 & 2013 & 2014 & 2015 & $p$ for \\
\hline$n$ & 426 & 1233 & 1822 & 2491 & 3111 & 3695 & 4211 & 4664 & 5067 & 5482 & 5650 & 5223 & 2115 & \\
\hline \multicolumn{15}{|l|}{ during the 1st year } \\
\hline $\begin{array}{l}\text { Age in the present } \\
\text { year, years; Mean (SD) }\end{array}$ & $\begin{array}{l}64.20 \\
(12.97)\end{array}$ & $\begin{array}{l}64.74 \\
(12.95)\end{array}$ & $\begin{array}{l}65.00 \\
(12.88)\end{array}$ & $\begin{array}{l}65.11 \\
(12.84)\end{array}$ & $\begin{array}{l}65.23 \\
(12.71)\end{array}$ & $\begin{array}{l}65.64 \\
(12.82)\end{array}$ & $\begin{array}{l}65.77 \\
(12.88)\end{array}$ & $\begin{array}{l}66.00 \\
(12.85)\end{array}$ & $\begin{array}{l}66.27 \\
(12.84)\end{array}$ & $\begin{array}{l}66.55 \\
(12.83)\end{array}$ & $\begin{array}{l}66.65 \\
(12.83)\end{array}$ & $\begin{array}{l}66.69 \\
(12.86)\end{array}$ & $\begin{array}{l}67.40 \\
(12.64)\end{array}$ & $<0.001$ \\
\hline $\begin{array}{l}\text { Period since AMI, } \\
\text { years; Mean (SD) }\end{array}$ & $\begin{array}{l}1.00 \\
(0)\end{array}$ & $\begin{array}{l}1.33 \\
(0.47)\end{array}$ & $\begin{array}{l}1.85 \\
(0.74)\end{array}$ & $\begin{array}{l}2.28 \\
(1.05)\end{array}$ & $\begin{array}{l}2.71 \\
(1.34)\end{array}$ & $\begin{array}{l}3.16 \\
(1.62)\end{array}$ & $\begin{array}{l}3.61 \\
(1.90)\end{array}$ & $\begin{array}{l}4.08 \\
(2.17)\end{array}$ & $\begin{array}{l}4.56 \\
(2.44)\end{array}$ & $\begin{array}{l}4.97 \\
(2.74)\end{array}$ & $\begin{array}{l}5.14 \\
(2.87)\end{array}$ & $\begin{array}{l}5.37 \\
(2.75)\end{array}$ & $\begin{array}{l}6.68 \\
(2.53)\end{array}$ & $<0.001$ \\
\hline
\end{tabular}

The data are presented as $\mathrm{n}(\%)$ unless otherwise stated. Dyslipidemia is defined as recorded diagnosis of dyslipidemia and/or average $\mathrm{LDL}$ level $>130 \mathrm{mg} / \mathrm{dL}$ during the hospitalization. Anemia is defined as recorded diagnosis of anemia and/or hemoglobin level $<14 \mathrm{~g} / \mathrm{dL}$ or hematocrit level $<42 \%$ for males and hemoglobin level $<12 \mathrm{~g} / \mathrm{dL}$ or hematocrit level $<37 \%$ for females during a hospitalization

Abbreviations: AMI Acute myocardial infarction; AV Atrioventricular; CABG Coronary artery bypass surgery; $C A D$ Coronary artery disease; $C H F$ Congestive heart failure; CIHD Chronic ischemic heart disease; COPD Chronic obstructive pulmonary disease; ED emergency department; GI Gastro-intestinal; ICCU Intensive Cardiac Care Unit; IHD Ischemic heart disease; LOS length of (hospital) stay; LM Left main (artery); LV Left ventricular; $P C I$ Percutaneous coronary intervention; PVD Peripheral vascular disease; SD standard deviation; STEMI ST segment elevation myocardial infarction; USD United States Dollars

\section{Discussion}

The current study investigated temporal trends in healthcare resource utilization throughout more than a decade (2003-2015) among post-AMI patients. Main findings include: throughout the investigated period utilization of most health-care resources, particularly the length of hospital stay and primary clinic visits have decreased. However, a major increase in ED visits was observed. The unadjusted healthcare expenditure has decreased throughout the study period. When this analysis is adjusted for potential confounders no statistically significant change in total cost was observed.

It has been widely described that AMI survivors are prone to increased morbidity (compared to matched general population), healthcare services utilization and economic burden which seem to be exceptionally high throughout the first year after the AMI followed by some subsequent decline $[2-5,16]$. We found a significant attenuation in the length of hospitalizations and ambulatory services utilization throughout the last decade yet an increase in the rate of ED visits and no change in total cost. This finding is consistent with the findings of Chen et al. [17] that showed a decrease in hospitalization of AMI patients (for heart failure) in Medicare beneficiaries 1998-2010 and with those of Chaudhry et al. [18] who reported a decline in the rate of recurrent AMI hospitalization between 1999 and 2000. Likosky et al. [9],who investigated readmission rate within the first year following AMI, found no difference throughout 1998-2008. Importantly, our analysis includes all hospitalizations rather than those for a particular cause, while previous reports showed that about $30 \%$ of readmissions are for reasons unrelated to the original condition [19]. In partial consistency with our findings, a recent report from the US found significant differences across the country and between different

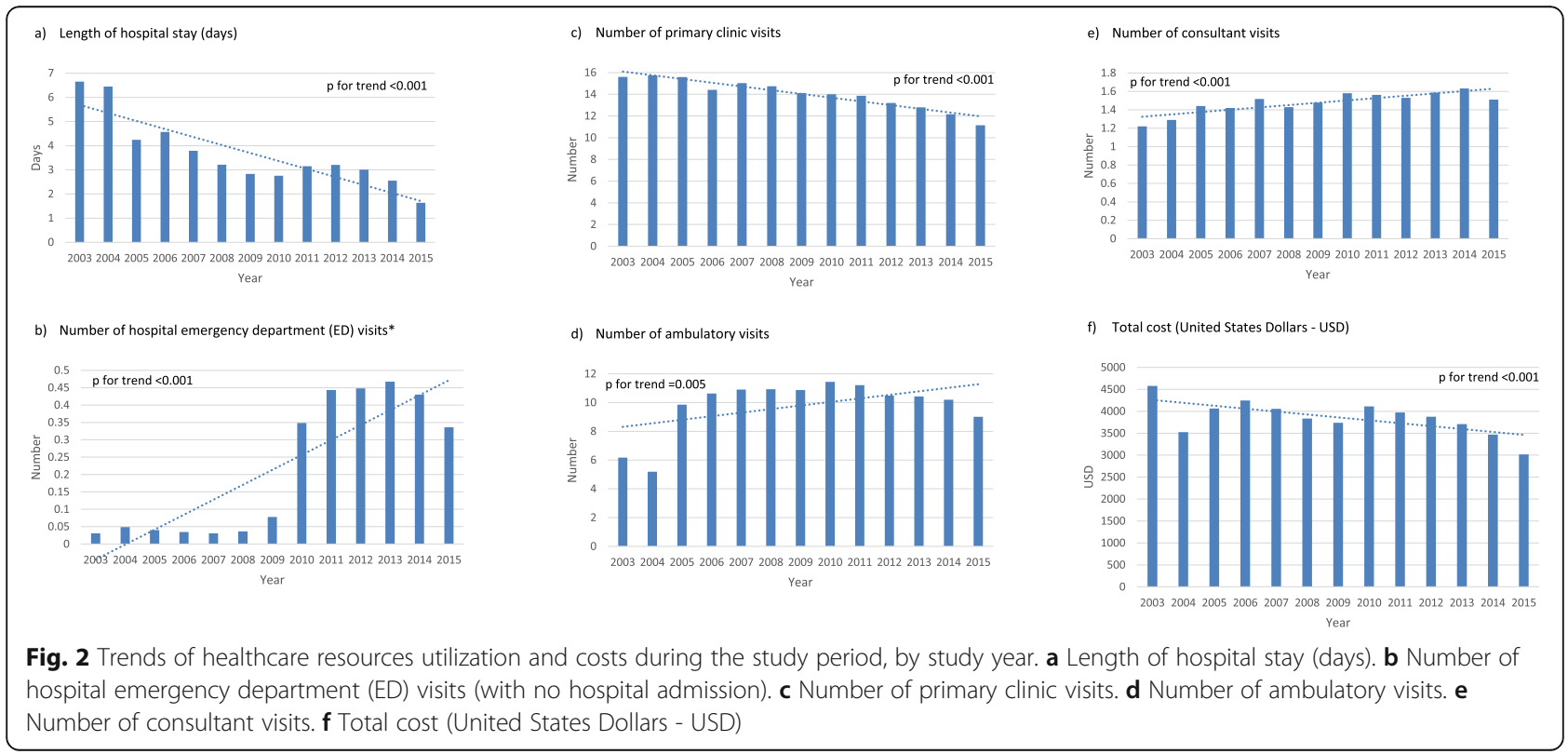


Table 2 Trends of healthcare resources utilization and costs - multivariate analysis

\begin{tabular}{|c|c|c|c|c|}
\hline Dependent variable & Model Level ${ }^{\mathrm{a}}$ & $B(S E)^{c}$ & $95 \% \mathrm{Cl}(\mathrm{B})$ & $\mathrm{p}$ \\
\hline \multirow[t]{3}{*}{ Length of hospital stay (days per patient-year) } & 1 & $-0.075(0.008)$ & $(-0.091 ;-0.060)$ & $<0.001$ \\
\hline & 2 & $-0.155(0.009)$ & $(-0.172 ;-0.137)$ & $<0.001$ \\
\hline & 3 & $-0.142(0.030)$ & $(-0.201 ;-0.083)$ & $<0.001$ \\
\hline \multirow[t]{3}{*}{ ED visits (number of visits per patient-year) ${ }^{b}$} & 1 & $0.276(0.009)$ & $(0.259 ; 0.293)$ & $<0.001$ \\
\hline & 2 & $0.277(0.021)$ & $(0.236 ; 0.318)$ & $<0.001$ \\
\hline & 3 & $0.289(0.014)$ & $(0.262 ; 0.317)$ & $<0.001$ \\
\hline \multirow[t]{3}{*}{ Primary clinic visits (number of visits per patient-year) } & 1 & $-0.358(0.026)$ & $(-0.409 ;-0.307)$ & $<0.001$ \\
\hline & 2 & $-0.696(0.026)$ & $(-0.746 ;-0.645)$ & $<0.001$ \\
\hline & 3 & $-0.612(0.025)$ & $(-0.661 ;-0.563)$ & $<0.001$ \\
\hline \multirow[t]{3}{*}{ Ambulatory visits (number of visits per patient-year) } & 1 & $0.009(0.004)$ & $(0.001 ; 0.018)$ & 0.033 \\
\hline & 2 & $-0.012(0.005)$ & $(-0.021 ;-0.003)$ & 0.008 \\
\hline & 3 & $0.008(0.005)$ & $(-0.001 ; 0.016)$ & 0.091 \\
\hline \multirow[t]{3}{*}{ Consultant visits (number of visits per patient-year) } & 1 & $0.015(0.003)$ & $(0.008 ; 0.021)$ & $<0.001$ \\
\hline & 2 & $-0.026(0.004)$ & $(-0.034 ;-0.019)$ & $<0.001$ \\
\hline & 3 & $-0.025(0.004)$ & $(-0.033 ;-0.017)$ & $<0.001$ \\
\hline \multirow[t]{3}{*}{ Total cost (USD per patient-year) } & 1 & $-0.015(0.005)$ & $(-0.025 ;-0.006)$ & 0.001 \\
\hline & 2 & $-0.050(0.006)$ & $(-0.061 ;-0.040)$ & $<0.001$ \\
\hline & 3 & $-0.005(0.011)$ & $(-0.027 ; 0.016)$ & 0.627 \\
\hline
\end{tabular}

${ }^{a}$ The first level models - unadjusted. The second level models - adjusted for the time following the Acute myocardial infarction (AMI). The third level models adjusted for the time following the AMI, age (at the evaluated year), sex, nationality, utilization / cost during an year before AMI, adherence to medical treatment during the 1st year, type of AMl, type of treatment and comorbidities (renal diseases, diabetes mellitus, neurological disorders, anemia, supraventricular arrhythmias, Congestive heart failure, Chronic obstructive pulmonary disease and Alcohol/drug addiction

${ }^{\mathrm{b}}$ Emergency department (ED) visits with no hospital admission

c Beta coefficient represents the degree of change in the outcome variable for every 1-unit of change in the predictor variable i.e. for every increase of 1 year throughout the follow-up period (e.g. 2003-2004 or 2014-2015) the LOS / cost differed by B units (day / USD), on average

Abbreviations: $B$ regression coefficient; $C l$ confidence interval; SE standard error; USD United States Dollars

services, in ambulatory and outpatient resource utilization and expenditure following hospital discharge with an AMI (31-180 days) between 1999 and 2014 [20].

The current study was not designed to determine the causes for the observed time dependent changes. However, several reasons can be suggested to explain the decline in hospitalizations and primary medicine services utilization. First and foremost, introduction of evidence based treatments in the acute phase management incorporated into AMI guidelines, improvements in the timeliness, and in particularly the use of invasive revascularization treatments as also observed in the current study $[21,22]$. Furthermore, significant improvements have been reported in secondary prevention treatments and post discharge management including control of risk factors, the use of guideline recommended medications and rehabilitation [18, 23]. Improved post-discharge adherence with guideline recommended therapies, as observed in the current study, is also an important factor which has previously been reported to be associated with reduced healthcare resources utilization [14]. It should be mentioned that individual long-term healthcare resource utilization tends to decrease after the first year post AMI [16].
However, this individual decline does not seem to be a prominent explanation for the observed trend in the current study, since an adjustment for the time from the index AMI (the second level models) was performed.

A trend of increase in ED visits was observed throughout the years, consistent with previous reports [20]. This disparity in the trends of ED utilization and the other resources could possibly be attributed to increased awareness and caution by the AMI survivors and their healthcare providers to complaints that could be related to an acute cardiac condition. Alternatively insufficient availability of outof-hospital services could all also cause increased frequency of ED visits [24]. Furthermore, the exceptionally greater increase in the ED visits overtime should be a focus of further investigation and intervention; for example, dedicated high quality out of hospital services with utilization of high sensitivity troponin point-of-care essays or the establishment of chest pain unit for such patient. In addition, a trend of increased age, increased prevalence of cardiovascular risk factors and other comorbidity were reported among AMI patients. The latter trends could counter-balance a trend of reduction and support a trend of increase in healthcare resources (e.g. ED visits) utilization [25, 26]. 
Total annual healthcare expenditure found in this study is lower than that reported in other studies [9-12]. This disagreement is probably due to differences in healthcare and insurance systems and the methodology for estimating healthcare expenditure. The unadjusted (the first level model) and the adjusted to time from AMI (the second level model), total cost has shown a significant trend of decrease throughout the evaluated decade. However, following adjustment to multiple potential confounders in a multivariate model (the third level model) we did not find statistically significant differences in overall healthcare expenditure throughout the investigated time period. The latter findings probably mean that the unadjusted decrease in costs is explained by the changes in patient characteristics, administered treatments (e.g. increase in interventional therapy) and improvement in adherence with medical therapy. A previous report, [9] found an increase of $16.5 \%$ in the healthcare expenditure per AMI patient between the years 1998-2008 in the US. Interestingly, the authors found that expenditures for skilled nursing facilities, hospice, home health agency, durable medical equipment, and outpatient care nearly doubled during the period of 31-365 days after admission. The differences can stem from different study periods, adjustment to confounders as well as differences in healthcare systems. A recent report that evaluated expenditure on Medicare beneficiaries 180 days following AMI in four time periods 1999-2000, 2004, 2008 and 2013-2014) found that expenditure increased 13.9\% from 1999 to 2000, and 2013,-2014, but declined 0.5\% between 2008 and 20132014 [20]. It should however be mentioned that excluding patients that died throughout the first year might have resulted in underestimation of costs since treating these patients is usually associated with increased expenditure [16]. This possibly had an impact over the temporal trend of costs, especially when considering the trend of improvement in short term survival observed during the study period [7].

Our findings should be discussed considering several important characteristics of the Israeli health care system. Israel has a national health insurance financed predominantly via progressive taxation and includes four competing, non-profit health plans (HPs) which provide a broad package of services determined by the government $[27,28]$. The HPs provide care in the community and purchase hospital services for their members. Nevertheless, approximately $40 \%$ of health care expenditure is financed privately. Access to primary care physicians and community-based specialists is good overall (travel and waiting times), although in some areas and populations (e.g. Israeli Arabs) it could be insufficient resulting in excessive use of hospital services. The occupancy rates of Israeli hospitals is one of the highest among the
Organization for Economic Co-operation and Development (OECD) countries (greater than 90\%) while the length of stay is one of the shortest (around 4 days) [27, 28]. Furthermore, hospital pricing system is considered outdated and limits their reimbursement while pricing of novel procedures and technologies and rising.

\section{Limitations}

The current study has several limitations. First, the study is retrospective and based mostly on administrative data (e.g. ICD-9-CM codes) hence prone for bias resulting from such a design such as recording bias. Furthermore, such a design also does not enable identification of the causes for the observed trends. Second, patients from only one hospital with unique characteristics, population mix and from a unique region in Israel were evaluated hence generalizability is limited. Third, differentiation of cardiovascular from non-cardiovascular related healthcare services utilization was not performed. Fourth, out of pocket expenses were not evaluated thus true costs are probably higher. Fifth, it is possible that unaccounted changes, not directly related to the AMI, in patients' health throughout the individual follow-up might have led to changes in health care utilization.

\section{Conclusions and implications}

The current study investigated temporal trends in healthcare resource utilization and costs in post-AMI patients throughout 2003-2015. The study shows a significant decrease in the length of the in-hospital stay and ambulatory services utilization with an increase in ED visits. Total costs (unadjusted or adjusted for the time following AMI), have declined. However, following adjustment for a variety of potential confounders no significant trend was found. Further and more comprehensive studies evaluating such trends, mechanisms and causes behind the observed findings, as well as the association between hospital services utilization and ambulatory utilization of healthcare services and costs in general and in subgroups of interest, are warranted, in order to improve resource allocation, patient outcomes and maximize cost-effectiveness in the longterm management of AMI patients. Nevertheless, even with current findings we believe that more resources and attention should be shifted towards ambulatory follow-up focusing on secondary prevention measures (i.e. optimal control of risk factors and prudent, cost-effective, early detection of subsequent and recurrent manifestations of atherosclerosis), particularly in high risk patients. Furthermore, we recommend reinforcing out of hospital measures for high quality chest pain evaluation such as a dedicated service, for chest pain evaluation (e.g. outpatient chest pain units) with high sensitivity troponin point-of-care essays and non-invasive coronary evaluation tests that would reduce ED visits and in-hospital evaluations. 


\section{Appendix}

\section{Appendix 1. Diagnoses and interventions according to ICD-9-CM codes}

Coronary artery bypass graft (CABG) Z361-Z362;

Thrombolytic therapy and/or Percutaneous coronary intervention (PCI): PCI Z3601-Z3607;

Renal diseases $403^{*}, 580-591^{*}, 7910, \mathrm{~V} 420,2504^{*}$;

Obesity 278, 2780\%;

Gastro-intestinal hemorrhage 53,021, 5307, 53,082, $5310^{*}, 5314^{*}, 5316^{*}, 5322^{*}, 5324^{*}, 5326^{*}, 5330^{*}, 5322^{*}$, $5334^{*}, 5336^{*}, 5340^{*}, 5342 \%, 5344^{*}, 5346^{*}, 53,501,53,511$, $53,521,53,531,53,541,53,551,53,561,53,783,53,784,56$, 202, 56,203, 56,212, 56,213,56,985, 578*;

Anemia 280-285\%;

Pulmonary heart disease $416^{*}-417^{*}$;

Chronic obstructive pulmonary disease $491^{*}, 492^{*}$, 494*, 496*;

Malignant neoplasm 148-208*, 230-233*;

Alcohol or drug addiction 303-3050\%, 3052-3059*, V113;

Neurological disorders 294\%, 310*, 320-349*, 430438*, Z0131*;

Schizophrenia and/or psychosis 295*, 298*, V110.

\section{Acknowledgements}

We are indebted to Mr. Asaf Cohen, Maccabi Institute for Health Services Research for his assistance on data preparation.

\section{Authors' contributions}

AS drafted the manuscript. HG and YP conceived the study, all authors participated in the design of the study, JEA and DG coordinated and helped in obtaining all the data, YP performed the data preparation and management and YP and AS analyzed the data. All authors read and approved the final manuscript.

\section{Funding}

The study was supported by the funds of Maccabi Institute for Health Services Research and The Israel National Institute for Health Policy research. The funding body had no role in the study design, data analysis and interpretation and writing the manuscript.

\section{Availability of data and materials}

Please contact author for data requests.

\section{Ethics approval and consent to participate}

The local ethics committees of the participating organizations and institutions approved the study, which was performed consistently with the Helsinki declaration. Receiving approval for informed consent was waived be the Helsinki committee.

\section{Consent for publication}

Not applicable.

\section{Competing interests}

The authors declare that they have no competing interests.

\section{Author details}

1 Department of Cardiology, Beilinson Hospital, Rabin Medical Center, Rabin Medical Center, 39 Jabotinski Street, 49100 Petah Tikva, Israel. ${ }^{2}$ Sackler Faculty of Medicine, Tel Aviv University, Tel Aviv, Israel. ${ }^{3}$ Faculty of Health Sciences, Ben-Gurion University of the Negev, Beer-Sheva, Israel. ${ }^{4}$ Maccabi Health Services, Southern Region, Beer-Sheva, Israel. ${ }^{5}$ Soroka University Medical Center, Beer-Sheva, Israel.
Received: 5 October 2019 Accepted: 29 January 2020

Published online: 12 February 2020

\section{References}

1. Plakht $\mathrm{Y}$, Gilutz $\mathrm{H}$, Shiyovich A. Excess long-term mortality among hospital survivors of acute myocardial infarction. Soroka acute myocardial infarction (SAMI) project. Public Health. 2017;143:25-36.

2. Yang E, Stokes M, Johansson S, Mellstrom C, Magnuson E, Cohen DJ, Hunt P. Clinical and economic outcomes among elderly myocardial infarction survivors in the United States. Cardiovasc Ther. 2016;34(6):450-9.

3. Janzon M, Henriksson M, Hasvold P, Hjelm H, Thuresson M, Jernberg T. Longterm resource use patterns and healthcare costs after myocardial infarction in a clinical practice setting: results from a contemporary nationwide registry study. Eur Heart J Qual Care Clin Outcomes. 2016;2(4):291-8.

4. Etemad LR, McCollam PL. Total first-year costs of acute coronary syndrome in a managed care setting. J Manag Care Pharm. 2005;11(4):300-6.

5. Zhao Z, Winget M. Economic burden of illness of acute coronary syndromes: medical and productivity costs. BMC Health Serv Res. 2011;11:35.

6. Menzin J, Korn JR, Cohen J, Lobo F, Zhang B, Friedman M, Neumann PJ. Relationship between glycemic control and diabetes-related hospital costs in patients with type 1 or type 2 diabetes mellitus. J Manag Care Pharm. 2010;16(4):264-75

7. Plakht Y, Gilutz H, Shiyovich A. Temporal trends in acute myocardial infarction: what about survival of hospital survivors? Disparities between STEMI \& NSTEMI remain. Soroka acute myocardial infarction II (SAMI-II) project. Int J Cardiol. 2016;203:1073-81.

8. Krumholz HM, Nuti SV, Downing NS, Normand SL, Wang Y. Mortality, hospitalizations, and expenditures for the Medicare population aged 65 years or older, 1999-2013. Jama. 2015;314(4):355-65.

9. Likosky DS, Zhou W, Malenka DJ, Borden WB, Nallamothu BK, Skinner JS. Growth in medicare expenditures for patients with acute myocardial infarction: a comparison of 1998 through 1999 and 2008. JAMA Intern Med. 2013:173(22):2055-61.

10. Plakht Y, Shiyovich A, Weitzman S, Fraser D, Zahger D, Gilutz H. A new risk score predicting 1- and 5-year mortality following acute myocardial infarction Soroka acute myocardial infarction (SAMI) project. Int J Cardiol. 2012:154(2):173-9.

11. Raebel MA, Schmittdiel J, Karter AJ, Konieczny JL, Steiner JF. Standardizing terminology and definitions of medication adherence and persistence in research employing electronic databases. Med Care. 2013;51(8 Suppl 3):S11-21.

12. Butler J, Arbogast PG, BeLue R, Daugherty J, Jain MK, Ray WA, Griffin MR Outpatient adherence to beta-blocker therapy after acute myocardial infarction. J Am Coll Cardiol. 2002;40(9):1589-95.

13. Hamood H, Hamood R, Green MS, Almog R. Determinants of adherence to evidence-based therapy after acute myocardial infarction. Eur J Prev Cardiol. 2016;23(9):975-85.

14. Bansilal S, Castellano JM, Garrido E, Wei HG, Freeman A, Spettell C, GarciaAlonso F, Lizano I, Arnold RJ, Rajda J, et al. Assessing the impact of medication adherence on long-term cardiovascular outcomes. J Am Coll Cardiol. 2016:68(8):789-801.

15. Choudhry NK, Glynn RJ, Avorn J, Lee JL, Brennan TA, Reisman L, Toscano M, Levin R, Matlin OS, Antman EM, et al. Untangling the relationship between medication adherence and post-myocardial infarction outcomes: medication adherence and clinical outcomes. Am Heart J. 2014;167(1):51-8 e55.

16. Plakht Y, Gilutz H, Arbelle JE, Greenberg D, Shiyovich A. Healthcare-service utilization and direct costs throughout ten years following acute myocardial infarction: Soroka acute myocardial infarction II (SAMI II) project. Curr Med Res Opin. 2019:1:1257.

17. Chen J, Hsieh AF, Dharmarajan K, Masoudi FA, Krumholz HM. National trends in heart failure hospitalization after acute myocardial infarction for Medicare beneficiaries: 1998-2010. Circulation. 2013:128(24):2577-84

18. Chaudhry SI, Khan RF, Chen J, Dharmarajan K, Dodson JA, Masoudi FA, Wang Y, Krumholz HM. National trends in recurrent AMI hospitalizations 1 year after acute myocardial infarction in Medicare beneficiaries: 1999-2010. J Am Heart Assoc. 2014;3(5):e001197.

19. Dunlay SM, Weston SA, Killian JM, Bell MR, Jaffe AS, Roger VL. Thirty-day rehospitalizations after acute myocardial infarction: a cohort study. Ann Intern Med. 2012;157(1):11-8

20. Likosky DS, Van Parys J, Zhou W, Borden WB, Weinstein MC, Skinner JS. Association between Medicare expenditure growth and mortality rates in 
patients with acute myocardial infarction: a comparison from 1999 through 2014. JAMA Cardiol. 2018;3(2):114-22.

21. Antman EM, Anbe DT, Armstrong PW, Bates ER, Green LA, Hand M, Hochman JS, Krumholz HM, Kushner FG, Lamas GA, et al. ACC/AHA guidelines for the management of patients with ST-elevation myocardial infarction--executive summary: a report of the American College of Cardiology/American Heart Association task force on practice guidelines (writing committee to revise the 1999 guidelines for the Management of Patients with Acute Myocardial Infarction). Circulation. 2004;110(5):588-636.

22. Peterson ED, Shah BR, Parsons L, Pollack CV Jr, French WJ, Canto JG, Gibson CM, Rogers WJ. Trends in quality of care for patients with acute myocardial infarction in the National Registry of myocardial infarction from 1990 to 2006. Am Heart J. 2008;156(6):1045-55.

23. Arbel Y, Matetzky S, Gavrielov-Yusim N, Shlezinger M, Keren G, Roth A, Kopel E, Finkelstein A, Banai S, Klempfner R, et al. Temporal trends in all-cause mortality of smokers versus non-smokers hospitalized with ST-segment elevation myocardial infarction. Int J Cardiol. 2014;176(1):171-6.

24. Nguyen JT, Vakil K, Adabag S, Westanmo A, Madlon-Kay R, Ishani A, Garcia S, McFalls EO. Hospital readmission rates following AMI: potential interventions to improve efficiency. South Med J. 2018;111(2):93-7.

25. Khot UN, Khot MB, Bajzer CT, Sapp SK, Ohman EM, Brener SJ, Ellis SG, Lincoff AM, Topol EJ. Prevalence of conventional risk factors in patients with coronary heart disease. Jama. 2003;290(7):898-904.

26. Plakht Y, Abu Eid A, Gilutz H, Shiyovich A. Trends of cardiovascular risk factors in patients with acute myocardial infarction: Soroka acute myocardial infarction II (SAMI II) project. Angiology. 2018:3319718816479:530.

27. Rosen B, Waitzberg R, Merkur S. Israel: health system review. Health Syst Trans. 2015;17(6):1-212

28. Waitzberg R, Quentin W, Daniels E, Perman V, Brammli-Greenberg S, Busse R, Greenberg D. The 2010 expansion of activity-based hospital payment in Israel: an evaluation of effects at the ward level. BMC Health Serv Res. 2019; 19(1):292

\section{Publisher's Note}

Springer Nature remains neutral with regard to jurisdictional claims in published maps and institutional affiliations.

Ready to submit your research? Choose BMC and benefit from:

- fast, convenient online submission

- thorough peer review by experienced researchers in your field

- rapid publication on acceptance

- support for research data, including large and complex data types

- gold Open Access which fosters wider collaboration and increased citations

- maximum visibility for your research: over $100 \mathrm{M}$ website views per year

At $\mathrm{BMC}$, research is always in progress.

Learn more biomedcentral.com/submissions 